\title{
Preliminary Study of End-Effector Compliance for Reducing Insertion Force in Automated Fluid Coupling for Trains
}

\author{
Kourosh Eshraghi ${ }^{a *}$, Pingfei Jiang ${ }^{a}$, Daniele Suraci $^{a}$, and Mark Atherton ${ }^{a}$ \\ ${ }^{a}$ Department of Mechanical and Aerospace Engineering, Brunel University London, Uxbridge, UK
}

\begin{abstract}
Robotic assembly of mating parts (peg-in-hole $(\mathrm{PiH})$ ) inevitably encounters misalignments. Although passive endeffector compliance is key to successful alignment during the assembly, the literature does not propose many solutions for large misalignments, which is relevant to applications such as compliance of a robot end-effector for train fluid servicing. The results from physical experiments indicate insertion forces that are too large for practical applications, even with small misalignments. This preliminary study applies a hybrid approach combining physical experiments and simulation modelling for large motion PiH coupling with end-effector compliance. This provides a platform for investigating insertion force during misaligned coupling. The simulation model contains configurable parameters for robot compliance and $\mathrm{PiH}$ friction which are informed by the physical experiment results. The many robot compliances are lumped as two torsional springs on the pitch and yaw motion axis of the robot arm model. The simulation model is then calibrated using the physical results without having to conduct further intensive physical experiments. The calibrated model represents the physical measurements to a satisfactory degree, however its performance can still be improved.
\end{abstract}

Keywords: Compliant end-effector, passive compliance, design of experiments, automated fluid coupling, train maintenance

\section{Introduction}

It is well documented that UK rail traffic will considerably increase by 2047 , requiring a commensurate increase in national passenger fleet of between 5,500 and 12,000 vehicles (Rolling Stock Strategy Steering Group, 2018). This increase will produce a comparative challenge for maintenance depots in terms of increasing their service capacity to keep trains available and reliable. There will also be an increasing demand for accurate service data and asset condition monitoring, vital for modernising maintenance, which will be challenging for manual-based servicing. Not only capacity limits of current manual labour will be stretched, but also the desirability of subjecting humans to such tasks in the 21 st century will continue to be scrutinised in terms of working conditions and safety aspects.

It is generally accepted that autonomous servicing could make a positive contribution to meeting the above demands as supported by, for example, an economic and technical feasibility study of a robotic autonomous system for train-fluid servicing (Atherton et al., 2019). However, it will be technically challenging for an autonomous system to match manual-based servicing in achieving effective mechanical connections common to many train maintenance tasks, which are performed under uncertain conditions 
outdoor of maintenance sheds. In such environments, autonomously identifying train parts and accurately positioning the end-effectors is very difficult for vision systems and thus misalignments are inevitable.

A lab-based research demonstrator robot has been built to physically investigate compliant end-effectors for the servicing of train-fluids. The goal of the project is to ensure effective and reliable mechanical connections of misaligned fluid ports using passively compliant end-effectors (Eshraghi et al., 2020). This initial "CyberFluids" system (see Fig. 1) includes the servicing of fuel, Controlled Emissions Toilets (CET) and wheel sand as typical applications. CyberFluids is a Cartesian, track-based robot that runs alongside an almost full-scale mock-up train carriage that has 2 fluid ports for investigating autonomous servicing. The track alongside the train carriage shown in Fig. 1 is the robot X-axis. The robot has 7 Degrees of Freedom (DoF) and provides 5-axis positioning for 3 end-effectors mounted on insertion arms (Z-axis). Two of the $\mathrm{Z}$-axis arms accommodate fluid couplers and the third is for gripping the relevant dust caps. The nominal size of each train port (and cap) corresponds to the typical 2-inch fuel port and 3-inch CET port. The cap gripper has an adjustable jaw to accommodate both cap sizes. On the train side, the 2 -inch port is fixed to a manual 5-axis, non-back-drivable positioning stage that is used to deterministically misalign the ports during experiments.

In current manual train fluid servicing operations, many different types of fluid couplers/ports are used in servicing the train fluids. For each type of port coupler different combinations of linear and rotary motions are typically required to make the coupling. Therefore, there is a need to adapt and standardise train fluid ports for automated fluid servicing. Dixon Ez-link cam and groove couplers (Dixon, 2020) were selected for this purpose, requiring only a linear insertion motion to reliably make a secure and sealed connection. This linear motion is also less demanding of the robot end-effector while potentially making the automated fluid servicing faster. The CyberFluids end-effector (Fig. 2) was designed to accommodate the Dixon coupler, actuate its cam-locks and provide some arbitrary compliance for misaligned insertions.

Reducing insertion forces during the fluid coupling will result in a much smoother and safer operation. Increasing the operating misalignment range of the end-effector will reduce requirements on the robot positioning system resulting in a simpler, more reliable, and cheaper system. However, as expected there is a trade-off to make as the increase of misalignments increases forces, and at some point it could result in complete failure to couple and perhaps damage to other assets. Focusing specifically on how to incorporate the compliance within the end-effector will enable retrofitting to a robot and contribute to a more resilient robotic workstation capability that is relevant to other RAS applications.

The literature does not provide clear guidelines on developing end-effectors with a large misalignment range. Hence in this preliminary study, physical and numerical experiments are performed to begin to characterise the performance of the CyberFluids large motion compliant end-effector. Physical experiments are conducted within a DoE framework in order to improve the performance of the existing end-effector. A subsequent DoE is implemented to obtain an enhanced simulation model that accounts for uncontrollable parameters affecting the robotic fluid coupling process. For example, since compliance of the CvberFluids robot is difficult to ascertain physically and also the friction coefficient between the mating parts is uncertain, estimates of these parameters are sought for the simulation model without having to conduct further physical experimentation. The resulting calibrated simulation model then becomes a platform upon which it is possible to test future compliant end-effectors without the need of further physical experimentation.

The remainder of the paper is structured as follows: related work regarding analysis and modelling methods of peg-in-hole $(\mathrm{PiH})$ insertion is presented in Section 2. Methodology for investigating endeffector performance and calibrating the simulation is presented in Section 3. Section 4 provides the results for both physical experiments and simulation modelling, plus a detailed comparison between them. Main insights from the results and limitations of the study are discussed in Section 5. The paper is concluded in Section 6. 


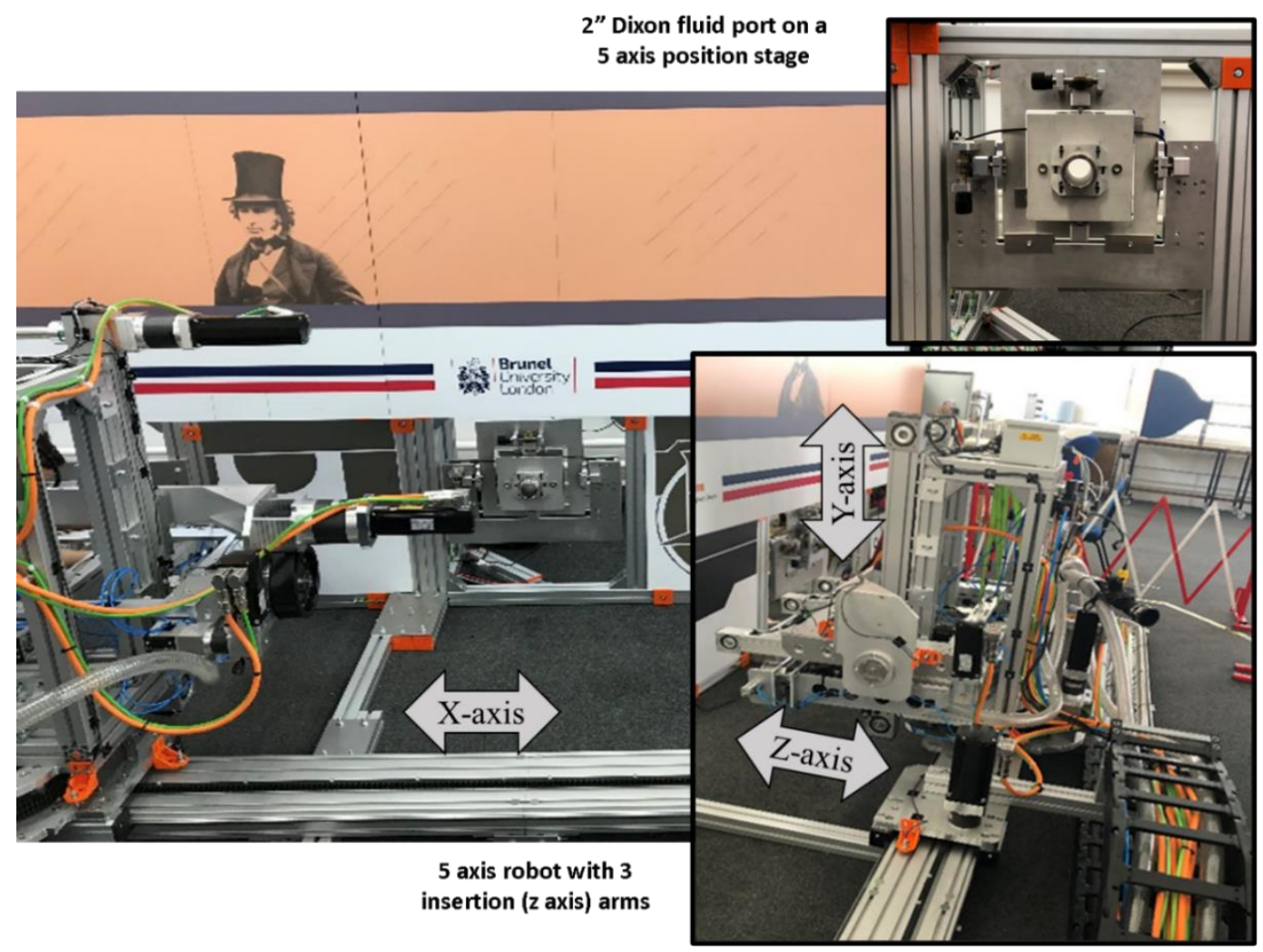

Fig. 1. CyberFluids train fluid servicing robot

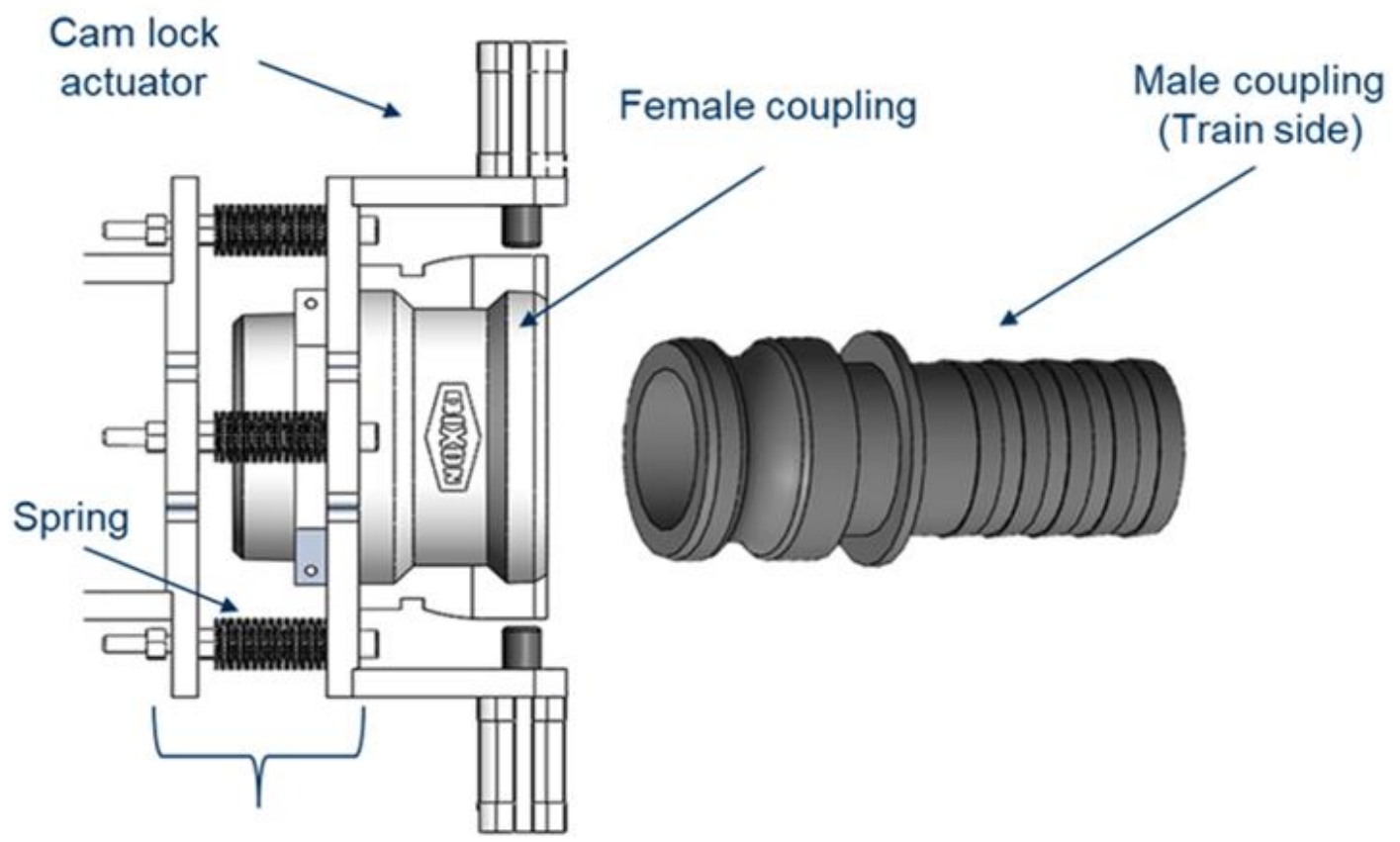

Compliant device

Fig. 2. Cyberfluid end-effector with Dixon couplers 


\section{Related Work}

In robotics, compliance is defined as the relationship between the motion and forces generated by a robot manipulator and an external part at their point of contact. Compliance can commonly be in the form of a spring-like stiffness, a generalised damper or a mechanical impedance that is used to accommodate and guide robot motions. Robot compliance can be categorised into active and passive compliance. Active compliance is achieved by controlling robot actuators and servo-motions ( $\mathrm{Xu}$ et al., 2019). Passive compliance is defined as intrinsic structural deflections such as flexibility of the robot base, limbs, joint transmissions and compliant end-effector (Wang et al., 1998). Active compliance reduces reliance on physical elements and corresponding complexity, and also aims at ensuring safety for humans. However, it also presents disadvantages such as power losses, relatively slow dynamic responses and reliance on sensors and control systems. Passive compliance makes a significant contribution to the development of more capable and robust robotics (Hawks \& Cutkosky, 2018). This is certainly the case in unstructured and outdoor applications where relying on active systems alone may be inefficient. Therefore, passive compliance is a logical consideration for a robotic system and is justification for further research to combine with developments in active compliance.

The fluid coupling operation is akin to robot assembly tasks that have a large body of research in which peg-in-hole $(\mathrm{PiH})$ analysis and robot compliance is key to successful manipulations. In assembly robotics, a PiH task refers to the process of automatically coupling of mating parts in order to make a fitting or assembly. The theory of robotic $\mathrm{PiH}$ originates from manufacturing assembly problems where most fittings are subjected to small engineering clearances. In such cases if the robot has no compliance even the smallest misalignments will cause large forces. Whitney et al (Whitney, 1982) developed an analytical 2-D PiH model for compliantly supported parts in manufacturing assembly applications. The compliances are modelled via linear springs that accommodate lateral (parallel) and angular misalignments. Initial rotational errors are neglected, and it is assumed the robot inserts in parallel to the hole axes. This gives rise to closedform solutions that can be used to identify jamming and wedging conditions where an estimate of the insertion force required. A passive compliance end-effector device known as the Remote Centre Compliance (RCC) is also recommended. By virtue of its elastic deformations and remote centre motion capabilities, this device will resolve contact forces to produce corrective motions that align the parts during insertion. A typical RCC device realises this special property through a geometrical arrangement of linkages that attain an instantaneous centre of rotation outside the physical envelope of the mechanism. In the literature, the RCC concept is only applied to small misalignments faced in manufacturing applications, performing properly within a range of $0.1 \mathrm{~mm}$. In a train maintenance environment, misalignments are in the order of $10 \mathrm{~mm}$, and thus outside of the normal scope of the RCC device and corresponding PiH theory. Furthermore, the assumptions of small angle and linear springs used in the $\mathrm{PiH}$ theory will not capture the nonlinear behaviour of large motion compliant mechanisms.

Since the work of Whitney, there has been much development in the PiH theory. Asada \& Kakumoto (1988) investigated high-speed insertions and developed the dynamic RCC (DRCC) and "no bounce" conditions, which Zohoor \& Shahinpoor (1991) addressed in further detail. Sturges and Laowattana (1996) investigated $\mathrm{PiH}$ in 3-dimensions and proposed the spatial RCC (SRCC) for polygon shaped parts. Other researchers have developed a Variable Remote Centre Compliance (VRCC) device which adapts to various peg lengths by adjusting its RCC projection point (Lee, 2000). Sathirakul \& Sturges (1998) considered multiple PiH insertion, which was an issue in the work of Kamnik et al (2001) for automation of battery lid assembly. Haskiya et al. (1998) developed chamfer-less PiH and a new type of compliance device based on the RCC (CVHRCC). Pitchandi et al. (2017) incorporated dashpot models to analytics of CVHRCC and studied the effects of damping during insertion. Spring elements and damper elements in SolidWorks motion studies were used to validate the analytical model. Jiang et al. (2017) employed PiH insertions for large aircraft components under small assembly misalignments. Wang et al. (2019) investigated the PiH issue for micro-assembly and developed a five DOF RCC based end-effector using Pseudo Rigid Body Modelling (PRBM). 
In summary, although the literature is extensive, there is a lack of studies that investigate large motion passive end-effectors for practical applications of largely misalignment $\mathrm{PiH}$ insertions. This could be due to:

(1). Limitations of small motion PiH theory that are confined to applications of precision manufacturing assembly and RCC devices.

(2). The influx of new literature on manipulator compliance and active methods for solving the PiH problem that risks overlooking the benefits of incorporating passive compliance in the end-effector.

(3). Lack of well-established methods for the design of compliant mechanisms for end-effectors.

Without clear guidelines in the literature, this study resorts to physical and numerical experimentation in order to improve and develop large misalignment PiH end-effectors.

\section{Research methodology}

A Design of Experiments (DoE) approach (Hinkelmann, 2012) is employed in order to maximise the chances of meaningful experimental results. It is often applied to systematically determine the relationship between system factors and their effect on the system output. System factors are generally categorised into controllable factors and noise factors, the former refers to factors that can be configured on purpose by the designer while the latter refers to factors that cannot be controlled in real situations, but they can often be configured in an experiment in order to serve the purposes of DoE. Output of the system, often referred to as system response, comprises one or more performance measures that quantitatively indicate how the system behaves upon factor variation. Typically, system responses are determined by regression functions that result in empirical system models that allow response prediction. These regression functions can exist in various formats such as first-order or second-order polynomial models, describing linear and quadratic behaviour of system responses respectively. Eq. (1) is a general expression of a second-order regression model. System response R can then be optimised towards a specific goal using the regression model obtained. The group of factor settings that satisfy the specific goal will be an optimal configuration for the system.

$$
R=\beta_{0}+\sum_{i=1}^{n} \beta_{i} x_{i}+\sum_{i=1}^{n} \beta_{i i} x_{i}^{2}+\sum_{i=1}^{n} \sum_{j=2}^{n} \beta_{i j} x_{i} x_{j}+\varepsilon
$$

$R$ represents system response, $n$ is the number of factors, $i$ and $j$ are the indices of each independent factor, $\beta$ are the coefficients for each independent term and $\varepsilon$ is an error term.

In this context, physical experiments only focus on improving existing designs hence, every time the design concept changes it requires further manufacturing and labour. Numerical approaches can be employed to rapidly develop and evaluate designs, this is particularly useful during the initial design stages. However, such models do not often capture the subtleties and imperfections encountered in physical reality. In this paper a numerical-physical hybrid approach is employed to calibrate simulations in which the effects of uncontrollable factors and other imperfections, are represented as lumped parameters. In the case of a robot performing $\mathrm{PiH}$ tasks, such noise factors typically revolve around unknown robot compliance and friction of mating parts, plus geometrical and material uncertainties. To gain a better understanding of the entire system, the initial physical experiments on the robot end-effector are conducted in a DoE framework. This provides more data on system behaviour which is used to:

A) increase the performance of the existing end-effector and

B) provide insight for the simulation calibration.

The benefit of this hybrid type approach lies in that a few physical experiments suffice for the development of a practical simulation model. As indicated in the flow chart of Fig. 3, this simulation environment then becomes a platform for developing other end-effector compliance designs. 
In this methodology section the existing design is first reviewed, followed by physical experiments performed on the robot assembly with DoE applied to systematically analyse the robot behaviours and the effect of end-effector design factors. Outcomes of this first analysis provide direct insight into how the new design can be steered towards better performance. In addition, these results from the physical experiments can also guide the construction of a simulation model using SolidWorks Motion Analysis (Dassault Systems, 2020). A second set of DoE are performed on the simulation model (a set of numerical experiments) in order to calibrate the model towards producing realistic results that can match with physical experiments. This calibrated model, which captures the subtleties and imperfections of a physical model with conveniences of a numerical model, provides an ideal platform for future end-effector analysis.

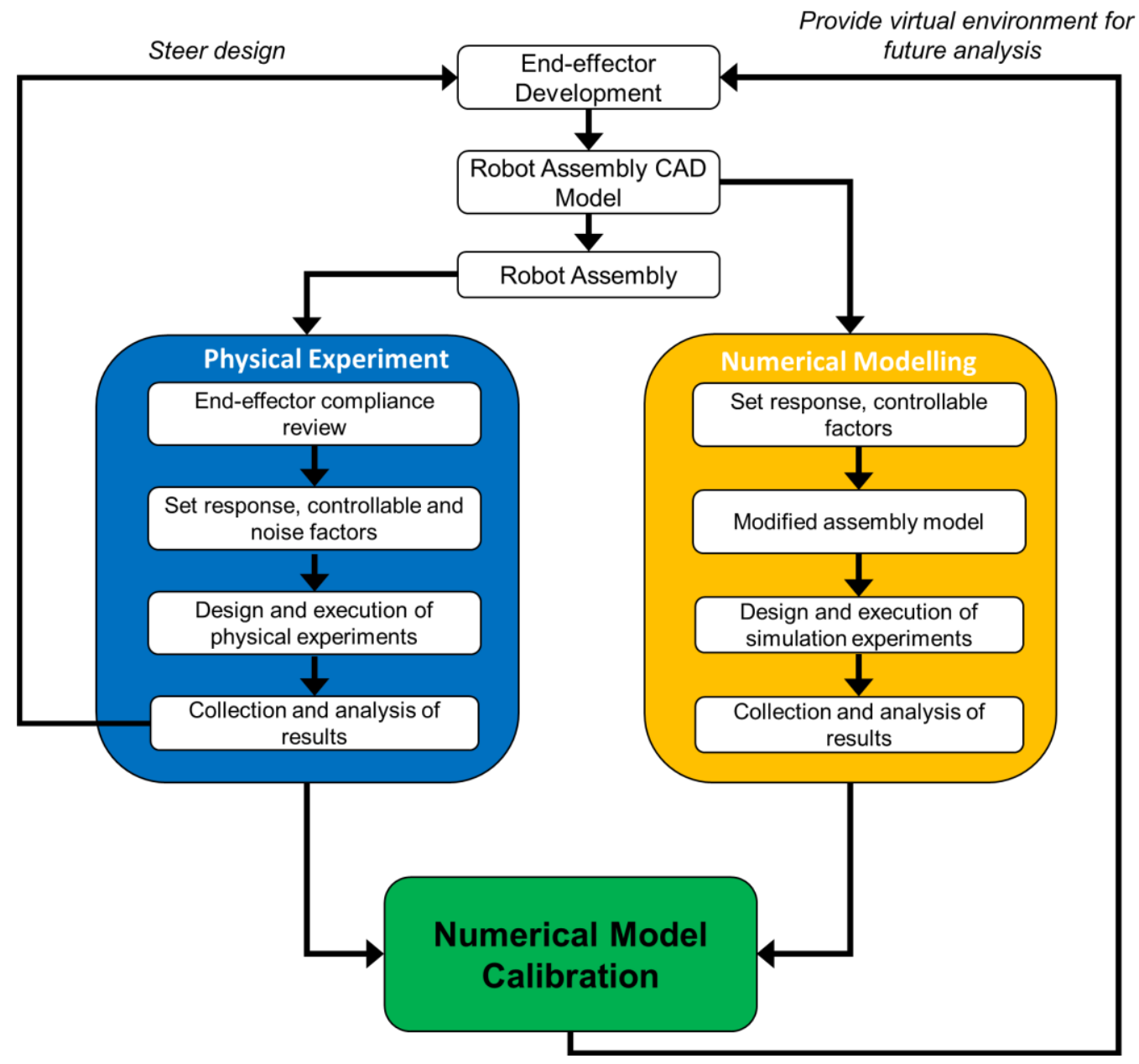

Fig. 3. Overview of the research methodology

\subsection{Physical experiments}

To select useful controllable factors for the physical experiments it is essential that a compliance review is conducted on the end-effector.

\subsubsection{End-effector compliance review}

As depicted in Fig. 2 and Fig. 4, physical end-effector compliance is facilitated by three springs that each encapsulate a shoulder bolt. The threaded part of the bolts is fixed to the coupler flange while the bolts are free to pivot and slide within corresponding clearance holes located in the arm flange. In this 
arrangement when all bolts slide simultaneously the end-effector compliance is linear in the $\mathrm{Z}$ direction. The clearance holes effectively act as spherical joints that have corresponding angular motions, which are amplified along the bolt length. The bolts are equally spaced around a pitch circle (radius, $P R$ ) that coincides with the centre of the coupler. Spring pre-compression ensures that the end-effector returns to its original position after a misaligned insertion. Maximum linear sliding in $\mathrm{Z}$ is determined by the compressed length of the springs. The coupler and its flange rotate and slide relative to the arm flange. The maximum rotation occurs when the bolts have two-point contact in the hole. Based on simple geometry a non-linear equation, Eq. (2), can be used to estimate this maximum angle.

$$
b \sin (\vartheta)+l \cos (\vartheta)=t
$$

Where $b$ is hole diameter, $l$ is hole length and $t$ is bolt diameter and $\theta$ is the maximum angle of rotation. The coupler has three DoF ( $\theta_{\mathrm{YZ}}, \theta_{\mathrm{XZ}}$ and $\left.\mathrm{Z}\right)$. As shown in Fig. 4, the $\mathrm{X}$ and $\mathrm{Y}$ linear motions of the coupler are coupled to the rotations in yaw $\left(\theta_{\mathrm{XZ}}\right)$ and pitch $\left(\theta_{\mathrm{YZ}}\right)$. Hole clearance can encourage small, non-elastic motions in the remaining directions ( $\mathrm{X} Y$ and $\theta_{\mathrm{XY}}$ ), however keeping the clearance to a minimum, these motions can be neglected as relatively small. The maximum range of linear motion is not symmetrical on either side of the X-axis i.e. in pitch motions. This is because bolts and springs either side of the X-axis have different moment arms, as shown in Fig. 4. This means that when the plate pivots the amplification effect will be different depending on the direction of motion. For similar reasons, compliance in the pitch axis is not symmetrical.
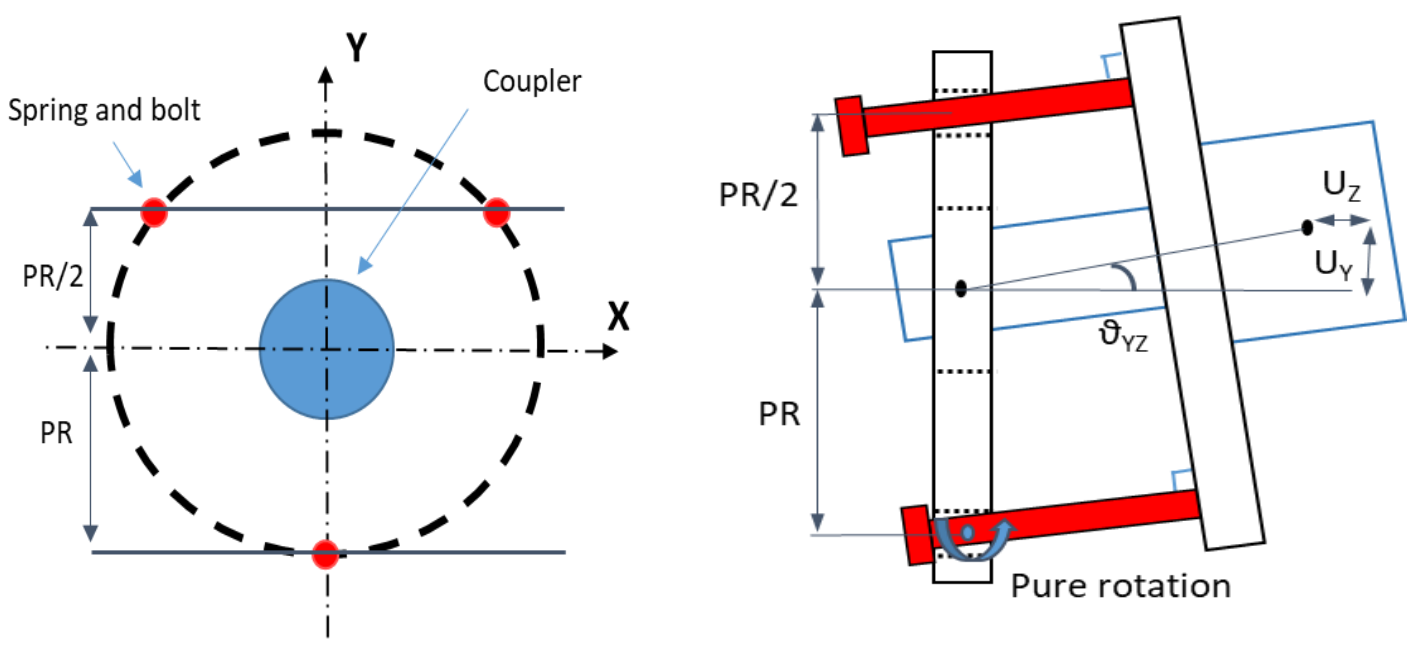

Fig. 4. Arrangement of CyberFluids end-effector

\subsubsection{Controllable factors: compliance}

The discussion above suggests spring stiffness, $K$, pitch radius $P R$ and the orientation of the set of holes, $O$, are potentially significant design factors that affect the end-effector compliance. Hole clearance will remain a constant and bolt length is not considered (or distance between the arm and coupler flanges) in order to reduce the number of variables (and experiments). Table 1 lists the experimental factors and Fig. 5 shows the modified experimental end-effector that can accommodate up to five adjustable levels for hole orientation, $O$, and pitch radius, $P R$. In order to easily adjust the spring stiffness, $K$, it was decided to use Belleville spring washers (see Fig. 6). A number of these spring washers can be stacked in parallel or series to achieve a large range of stiffness, deformation or load characteristics (Shigley, 2006). The selection of Bellville springs is not only constrained by the required range of stiffness or deformation, but also by bolt diameter. If the clearance between the washer and bolt is too large, even when the spring is compressed, 
washers can slide in the radial direction. This is undesirable as washers will not make contact at consistent points and this will cause an indeterminate change in spring parameters that will induce experimental error. It is also crucial to prevent the washers from jamming in the screw thread. Thus, bolt length, $L$, is selected to accommodate the longest spring washer stack. For spring arrangements with a lower free length, standard spacer washers are included in the stack to fill the remainder of the bolt length. All springs arrangements are pre-compressed to $15 \%$ of the total stack deformation (lower bound spring operating range recommended in DIN2093). As shown in Fig. 7, the selected spring has a nonlinear force-displacement relationship, thus:

$$
\frac{d f}{d x} \neq \text { Constant } K
$$

Table 1 Physical experimental parameters

\begin{tabular}{lcccc}
\hline \hline \multicolumn{1}{c}{ Parameters } & Nomenclature & Nominal Value & Experimental Range & Units \\
\hline Spring Eq. Stiffness & $\mathrm{K}$ & 4.6 & 3.2 to 6 & $\mathrm{~N} / \mathrm{mm}$ \\
Pitch Radius & $\mathrm{PR}$ & 91.5 & 65 to 118 & $\mathrm{~mm}$ \\
Bore Orientation & $\mathrm{O}$ & 0 & -25 to +25 & $\mathrm{0}$ \\
Bolt Length & $\mathrm{L}$ & 110 & Constant & $\mathrm{mm}$ \\
Arm/coupler Distance & $\mathrm{D}$ & 80 & Constant & $\mathrm{mm}$ \\
Bore Clearance & $\mathrm{C}$ & 0.5 & Constant & $\mathrm{mm}$ \\
Coupler/port Clearance & $\mathrm{CC}$ & 0.7 & Constant & $\mathrm{mm}$ \\
X-axis Misalignment & $\mathrm{XM}$ & $\approx 0$ & -5 to 5 & $\mathrm{~mm}$ \\
Y axis Misalignment & $\mathrm{YM}$ & $\approx 0$ & -5 to 5 & $\mathrm{~mm}$ \\
\hline \hline
\end{tabular}

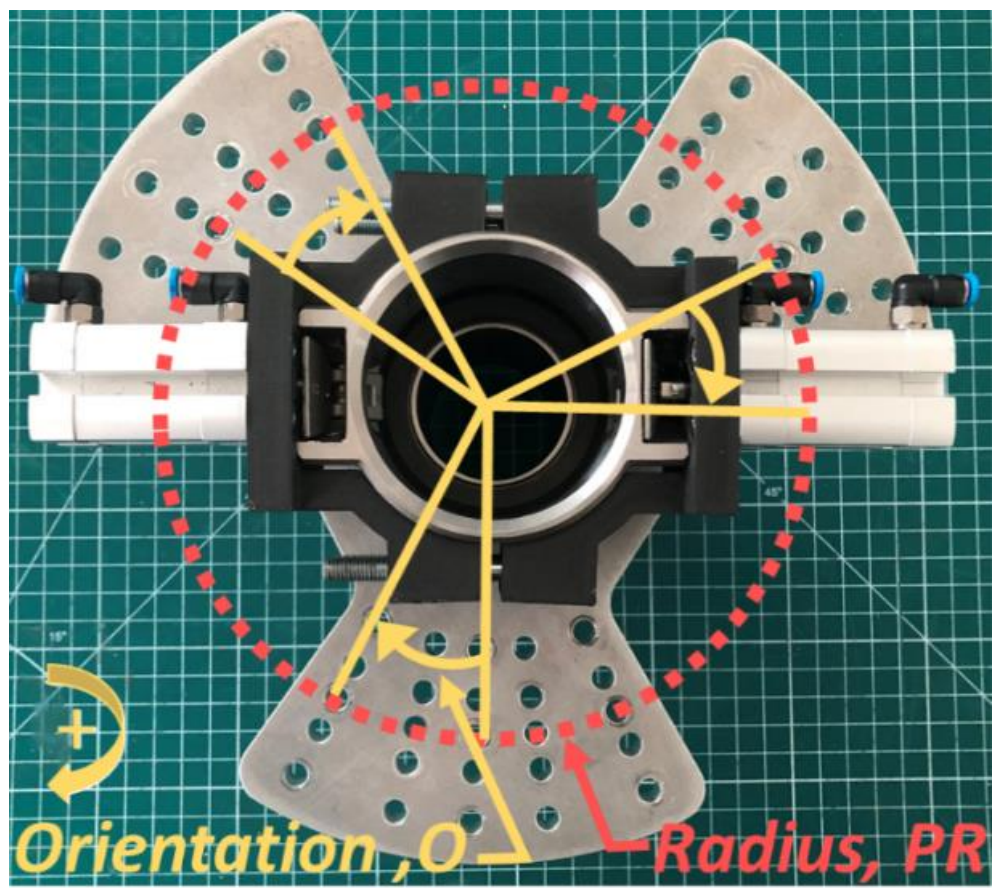

Fig. 5. Experimental end-effector 


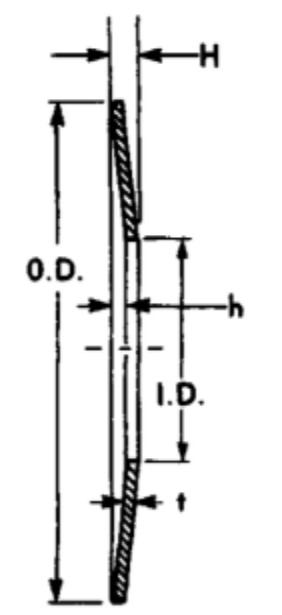

\begin{tabular}{cc}
\hline $\begin{array}{c}\text { Spring } \\
\text { Parameter }\end{array}$ & $\begin{array}{c}\text { Value } \\
\text { (mm) }\end{array}$ \\
\hline $\mathrm{H}$ & 0.8 \\
$\mathrm{~h}$ & 0.45 \\
$\mathrm{t}$ & 0.35 \\
O.D & 12.5 \\
I.D & 6.2 \\
\hline
\end{tabular}

Fig. 6. Parameters of the selected Belleville spring washer (Shigley, 2006)

In order to derive a single parameter that serves as the stiffness constant (design parameter, $K$ ), the forcedisplacement relationship shown in Fig. 7 is linearised. Since the relationship between the number of washers and deformation/load is linear, the regression fit is independent of the number of washers stacked. With $R^{2}=0.898$ and $p$-Value $=0.00404$, the regression model has a good fit. Hence linearisation is a simple and reliable method of comparing the stiffness of various washer stack arrangements. Eq. (4) is used to identify the number of washers (in series) that will deliver the required linear stiffness.

$$
N_{\text {series }}=\left(\frac{\text { Linear Stiffness of } 1 \text { washer }}{\text { Required Linear Stiffness }}\right)^{\text {Integer }}
$$

The number of washers must obviously be a discrete value and thus, due to rounding up/down, there are errors in achieving the exact required linear stiffness. However, if a large number of washers are stacked in series this error becomes very small. Using the selected spring, for a range of 3.2 to $6 \mathrm{~N} / \mathrm{mm}$ (91 to 49 spring washers) maximum error in the linear stiffness is $0.064 \%$.

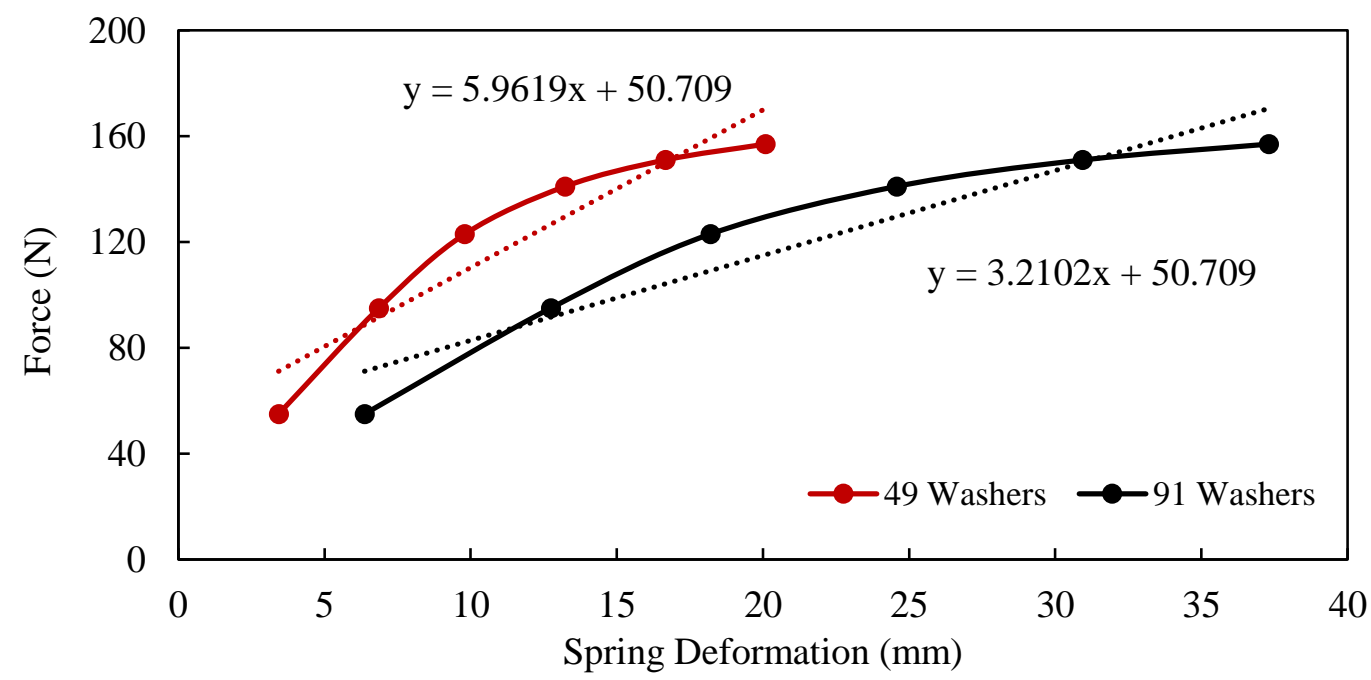

Fig. 7. Force displacement relationship of the selected Belleville washers 


\subsubsection{Noise factors: misalignments}

For an XYZ (three DoF) Cartesian RAS discussed by Atherton et al (2019), robot X and Y-axis misalignments lead to poor coupling. Axial misalignments in $\mathrm{Z}$ (insertion direction) only contribute to the coupling seal and hence are neglected in this study. For these experiments train fluid ports are aligned parallel to the robot Z-axis and the CyberFluids robot will only use XYZ motion to make the fit. Initial experiments indicated that ports do not couple satisfactorily when $\mathrm{X}$ and $\mathrm{Y}$ axes are misaligned more than $5 \mathrm{~mm}$. Hence, the experimental range of misalignments is selected accordingly. Misalignments are measured using Vernier callipers from a fixed datum on the train fluid port positioning stage.

\subsubsection{Response: motor readings}

A perfectly aligned fluid coupling is expected to couple with minimal force; a high insertion force will indicate high friction and/or physical clash that will occur due to misalignment between the coupler and fluid port. Therefore, considering the complete insertion cycle, we can use the energy quantity, work done by the motor, as a scalar measure of coupling performance. Insertion force is also monitored, as this is important for sizing actuators, robot structure and preventing damage to the robot or train parts.

Robot servo-drives can monitor motor current and position, which is used to determine insertion force and linear position of the end-effector arm. The relationship between motor current and torque is linear and defined by a motor torque constant specified by the manufacturer. In order to obtain work done, torque must be converted to force and integrated over the linear distance travelled.

$$
\begin{gathered}
\text { Motor Torque, } \mathrm{T}_{\mathrm{M}}=\mathrm{k}_{\mathrm{M}} * \mathrm{I}_{\mathrm{rms}} \\
\text { Gearbox Torque, } \mathrm{T}_{\mathrm{G}}=\mathrm{T}_{\mathrm{M}} * 5 \\
\text { Insertion Force, } \mathrm{F}_{\mathrm{z}}=\mathrm{T}_{\mathrm{G}} / \mathrm{r} \\
\text { Work }, \mathrm{W}=\int F_{\mathrm{Z}} d_{Z}
\end{gathered}
$$

Where $k_{M}$ is the motor torque constant, $I_{R M S}$ is the root mean square of the 'torque generating' current and $r$ is the radius of the pulley drive in the insertion arm.

The servo-drive is capable of recording 200 samples for motor position and current. The robot insertion speed was set to a nominal value of $25 \mathrm{~mm} / \mathrm{s}$, and a sampling rate of $20 \mathrm{~ms}$ was used in order to capture the entire event of coupling with sufficient precision.

\subsubsection{Experiment plan}

Face Central Composite Design (FCCD) of experiments (Montgomery, 2012) is used to investigate the influence of robot end-effector design factors on the resultant energy consumption to perform an effective coupling. An FCCD for five factors $K, P R, O, X M$ and $Y M$ requires 36 experiments with each factor configured at three levels: low, medium and high. These levels are generally codified as $-1,0$ and 1 respectively. Actual settings for each factor were interpolated by referring to their actual limits. Table 3 shows the experimental plan in actual values using FCCD with a revised order to minimise human effort in changing end-effector configurations. Furthermore, centre runs were performed at different points during the experiments in order to effectively capture more of the experimental errors. 


\subsection{Simulation modelling}

SolidWorks Motion Analysis is a tool for modelling rigid body kinematics/dynamics with contact and friction in mechanical assemblies (Motion Analysis Overview, 2020). It also provides the option of adding springs, dampers and custom forces which can be used to model compliance between parts or assembly mates.

A second DoE is planned with the aim of enhancing SolidWorks Motion Analysis simulations, representative of the robotic fluid coupling process. DoE is applied here to identify the best configuration of settings that can provide the most reliable predictions of end-effector performances when compared to the physical experiments.

\subsubsection{Controllable factors: friction and compliance}

Using rigid groups and SolidWork's primitive mates, the whole robot assembly is simplified to reduce components and eliminate redundant degree of constraint (DoC). A new $2 \mathrm{R}$ joint is added at an arbitrary position on the simplified robot model (Fig. 8). Coupled to torsional springs this new joint represents the unknown compliance behaviour of the robot and including the friction of mating parts (fluid ports), three factors are identified, namely static friction $\left(F_{s}\right)$, robot compliance in pitch $(P K)$ and robot compliance in yaw $(Y K)$. The nominal values and experimental range of these parameters are presented in Table 2 . It should be noted that kinetic friction coefficient $\left(F_{k}\right)$ is set to $F_{k}=F_{s}-0.05$.

Initial simulations show that coupling fails when using SolidWorks steel friction parameters $\left(F_{s}=0.3\right.$, $\left.F_{k}=0.25\right)$. Therefore, a lower range is selected for this parameter and those selected for compliance of pitch and yaw are based on experience from conducting the physical experiments. A summary of the controllable factors for the simulation can be found in Table 2 .

Table 2 SolidWorks Motion Analysis controllable factor setup

\begin{tabular}{lllll}
\hline Controllable Factors & Nomenclature & Nominal Value & Experimental Range & Units \\
\hline Static Friction Coef. & $\mathrm{F}_{\mathrm{s}}$ & 0.16 & 0.06 to 0.26 & $\mathrm{~N} / \mathrm{A}$ \\
Robot compliance in Pitch direction & $\mathrm{PK}$ & 350 & 150 to 550 & $\mathrm{KNm} /$ degree \\
Robot compliance in Yaw direction & $\mathrm{YK}$ & 350 & 150 to 550 & $\mathrm{KNm} /$ degree \\
\hline \hline
\end{tabular}

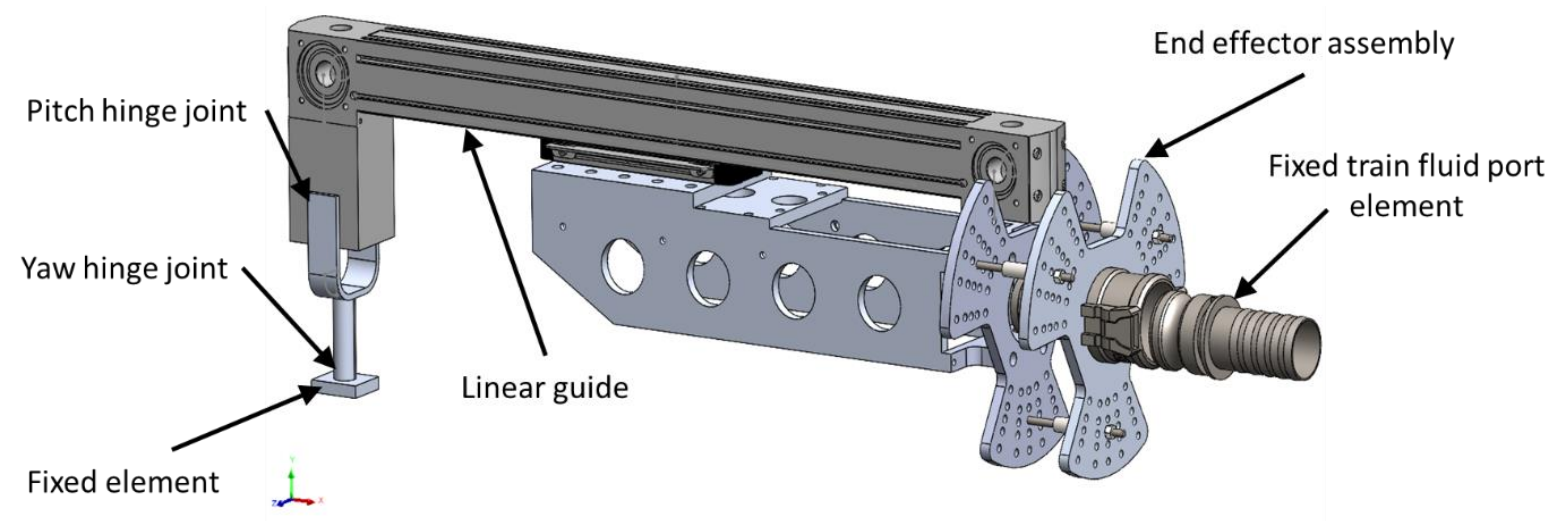

Fig. 8. Simplified robot arm assembly for SolidWorks Motion Analysis 


\subsubsection{Response: plots}

As in the physical experiments, force required to drive the motor is the main output from the simulations. Force can be plotted against time or motor displacement to determine peak value and work done which again is determined using the same formulations of Section 3.1.3.

\subsubsection{Experiment plan}

Initial setup simulations indicated a 30-minute period required per experimental run hence reducing the number of experiments while maintaining the capability to model quadratic behaviours is the primary consideration when choosing the DoE method.

A full second order regression model of three factors has 10 terms, covering intercept, first order term, second order term and interactions terms. In DoE, in order to estimate the coefficient of one term, a minimum of one experiment run is required, meaning at least 10 runs are needed for estimating all the coefficients. However, typically three addition runs are added to estimate the model error term $\varepsilon$. As a result, 13 runs are configured using D-Optimal design (Montgomery, 2012).

The physical experiments have 27 unique combinations (36 minus 9 from the 10 repeated centre runs), in order to fully estimate the outcome of the simulation, the $13 \mathrm{D}$-Optimal runs need to be applied to each 27 physical experiments, resulting in $13 * 27=351$ simulations. This is simply not feasible considering the time required for each simulation. As a result, the strategy for calibration of the simulation model is that the 13 D-Optimal runs are applied to one physical experiment first in order to identify candidate friction, pitch and yaw compliance settings. The obtained optimal setting is then validated through three more physical experiments. In this case only a total number of 16 simulations are required.

\subsubsection{Motion analysis elements setup}

Following are the SolidWorks Motion Analysis "elements" used to simulate the robotic fluid coupling systems.

(1). Torsional springs: To imitate compliance, new hinges are added on the robot arm model. Linear torsional springs with stiffness values suggested by the DoE are designated to these hinges.

(2). Forces

- Springs: The standard motion analysis spring element does not accurately model our nonlinear Belleville springs. To overcome this issue, force elements have been used instead. Using the Belleville spring design equation, the spring force-displacement relationship is expressed as Eq.(9) (Shigley, 2006). The solid length of the springs is represented by rigid sleeves placed on the bolts.

$$
\begin{gathered}
P=\frac{E f}{\left(1-\mu^{2}\right) M a^{2}}\left[(h-f)\left(h-\frac{f}{2}\right) t+t^{3}\right] \\
M=\frac{6}{\pi \ln (R)}\left(\frac{(R-1)^{2}}{R^{2}}\right)
\end{gathered}
$$

Where, $P$ is load, $E$ is the elastic modulus, $f$ is the deformation, $\mu$ is the Poisons ratio, $a$ is the $O D / 2, t$ is the thickness of the washer, $h$ is the information available before the washer cone flattens, $R=O D / I D$

- Forces (drive friction): from the physical results it was clear that a constant driving force of $43 \mathrm{~N}$ is required to maintain a robot arm speed of $25 \mathrm{~m} / \mathrm{s}$. This force was included in the simulations.

(3). Linear motor: The speed is set to a constant value of $25 \mathrm{~mm} / \mathrm{s}$, which should act similar to the robot servo.

(4). Solid body contacts: there are essentially two regions where contact is expected. 
- First contact zone is between the fluid ports that are being coupled, the material is set to stainless steel hence the typical elastic and impact properties apply. However, the static and kinetic coefficients of frictions are varied as suggested by the DoE.

- Second contact zone is between the bolts and the robot end-of-arm flange. Since the bolts are stainless steel and the end-of-arm flange is aluminium, all properties are set accordingly.

\subsubsection{Motion analysis settings}

(1). Frames per second (fps): is set to $50 \mathrm{fps}$ to ensure results are obtained as per $20 \mathrm{~ms}$, which is the sampling rate used to obtain physical results.

(2). Contact resolution: is set to "precise contact" with an integrator accuracy of 0.0001 to ensure analytically sound results

(3). Advanced options: are kept to default settings that use a variable order, variable step size integrator.

\section{Results}

The results are categorised into two sections:

○ Results and analysis of the physical experiments, an attempt on end-effector performance improvement.

- Results from the simulation modelling which enables the calibration of the simulation model.

All DoE related tables show the actual values of settings for ease of understanding. All regression analysis is performed using coded values for consistency.

\subsection{Physical experiment results}

Results for the physical experiments are shown in Table 3. A key finding from the results of the physical experiments is the significance of the regression model (Table 5) with $P R$ and $K$ being the parameters of considerable effect, see the significance of these parameters in Table 5. Generally, softer springs and lower values of $P R$ results in lower forces and work done. Fig. 9 shows the mean effect of the significant factors in terms of the observed work done. By averaging, the observed responses when each factor was at its higher or lower limits, the typical effect of a design factor is determined (Atherton \& Bates, 2006).

Obviously, coupling with large misalignments should produce a higher value of work done. However, mean response is significantly lower in the $-Y$ and $+X$ directions. This is understandable for the $Y$-direction as end-effector pitch compliances are not symmetrical. It is interesting to have observed this in the $\mathrm{X}$ direction as this could indicate the preferred directions of compliance (end-effector, robot and fluid port) in the system. This phenomenon and the lack of symmetry is evident throughout the results. 
Table 3 Physical experiment plan and results

\begin{tabular}{llllllllllllllll}
\hline \hline Order & $\mathrm{K}$ & $\mathrm{PR}$ & $\mathrm{O}$ & $\mathrm{XM}$ & $\mathrm{YM}$ & $\begin{array}{l}\text { Work } \\
(\mathrm{Nm})\end{array}$ & $\begin{array}{l}\text { Max } \\
\text { Force } \\
(\mathrm{N})\end{array}$ & Order & $\mathrm{K}$ & $\mathrm{PR}$ & $\mathrm{O}$ & $\mathrm{XM}$ & $\mathrm{YM}$ & Work(Nm) & $\begin{array}{l}\text { Max } \\
\text { Force } \\
(\mathrm{N})\end{array}$ \\
\hline 1 & 4.6 & 91.5 & 0 & 0 & 0 & 1.89 & 49.64 & 19 & 6 & 65 & -25 & -5 & -5 & 4.20 & 329.85 \\
2 & 4.6 & 91.5 & 0 & 0 & 0 & 2.03 & 60.85 & 20 & 6 & 65 & -25 & 5 & 5 & 6.05 & 521.99 \\
3 & 4.6 & 91.5 & 0 & 0 & 0 & 1.94 & 59.24 & 21 & 6 & 65 & 25 & -5 & 5 & 8.92 & 611.66 \\
4 & 4.6 & 91.5 & 0 & 0 & 0 & 1.89 & 51.24 & 22 & 6 & 65 & 25 & 5 & -5 & 3.42 & 209.76 \\
5 & 4.6 & 91.5 & 0 & 0 & 0 & 1.89 & 49.64 & 23 & 3.2 & 65 & 25 & -5 & -5 & 3.73 & 285.01 \\
6 & 4.6 & 91.5 & 0 & -5 & 0 & 4.92 & 414.71 & 24 & 3.2 & 65 & 25 & 5 & 5 & 7.46 & 542.81 \\
7 & 4.6 & 91.5 & 0 & 0 & 0 & 1.98 & 60.85 & 25 & 3.2 & 118 & -25 & -5 & -5 & 4.30 & 344.26 \\
8 & 4.6 & 91.5 & 0 & 0 & 0 & 1.89 & 48.04 & 26 & 3.2 & 118 & -25 & 5 & 5 & 10.78 & 643.68 \\
9 & 4.6 & 91.5 & 0 & 5 & 0 & 3.04 & 163.32 & 27 & 6 & 118 & -25 & -5 & 5 & 11.13 & 648.49 \\
10 & 4.6 & 91.5 & 0 & 0 & -5 & 2.66 & 118.49 & 28 & 6 & 118 & -25 & 5 & -5 & 3.86 & 291.42 \\
11 & 4.6 & 91.5 & 0 & 0 & 5 & 5.67 & 493.17 & 29 & 6 & 118 & 25 & -5 & -5 & 9.34 & 630.87 \\
12 & 3.2 & 91.5 & 0 & 0 & 0 & 2.04 & 60.85 & 30 & 6 & 118 & 25 & 5 & 5 & 9.03 & 650.09 \\
13 & 6 & 91.5 & 0 & 0 & 0 & 2.06 & 67.25 & 31 & 3.2 & 118 & 25 & -5 & 5 & 9.30 & 570.03 \\
14 & 4.6 & 91.5 & -25 & 0 & 0 & 2.01 & 60.85 & 32 & 3.2 & 118 & 25 & 5 & -5 & 3.68 & 257.79 \\
15 & 4.6 & 91.5 & 25 & 0 & 0 & 2.17 & 76.86 & 33 & 4.6 & 118 & 0 & 0 & 0 & 2.06 & 68.85 \\
16 & 4.6 & 65 & 0 & 0 & 0 & 2.08 & 73.66 & 34 & 4.6 & 91.5 & 0 & 0 & 0 & 2.09 & 73.66 \\
17 & 3.2 & 65 & -25 & -5 & 5 & 9.32 & 611.66 & 35 & 4.6 & 91.5 & 0 & 0 & 0 & 1.96 & 59.24 \\
18 & 3.2 & 65 & -25 & 5 & -5 & 3.41 & 261.00 & 36 & 4.6 & 91.5 & 0 & 0 & 0 & 1.95 & 57.64 \\
\hline \hline
\end{tabular}

Table 4 Statistics of fitted regression model from the physical experiment

\begin{tabular}{llllll}
\hline \hline Model & $\mathrm{p}$-value & $\mathrm{R}^{2}$ & Adjusted R & Root Mean Square Error & F-statistic \\
\hline Work $_{\text {physical }}$ & $5.95 \mathrm{e}^{-12}$ & 0.991 & 0.979 & 0.429 & 84.6 \\
Max-Force $_{\text {physical }}$ & $01.45 \mathrm{e}^{-10}$ & 0.986 & 0.968 & 40.7 & 54.7 \\
\hline \hline
\end{tabular}



coupling for trains

Table 5 Statistics of fitted regression model terms

\begin{tabular}{lcccc}
\hline \hline & \multicolumn{2}{c}{ Work physical } & \multicolumn{2}{c}{ Max-Forcephysical } \\
& $\boldsymbol{\beta}$ & p-Value & $\boldsymbol{\beta}$ & p-Value \\
\hline (Intercept) & 1.932 & 0.000 & 67.366 & 0.000 \\
K & 0.221 & 0.045 & 21.349 & 0.042 \\
PR & 0.827 & 0.000 & 36.561 & 0.002 \\
O & 0.111 & 0.290 & 6.761 & 0.492 \\
XM & -0.801 & 0.000 & -50.260 & 0.000 \\
YM & 2.170 & 0.000 & 142.507 & 0.000 \\
$\mathrm{~K}^{\wedge} 2$ & 0.141 & 0.615 & -16.272 & 0.539 \\
$\mathrm{PR}^{\wedge} 2$ & 0.163 & 0.560 & -9.066 & 0.731 \\
$\mathrm{O}^{\wedge}$ & 0.182 & 0.516 & -11.468 & 0.664 \\
$\mathrm{XM}^{\wedge} 2$ & 2.072 & 0.000 & 208.697 & 0.000 \\
$\mathrm{YM}^{\wedge} 2$ & 2.254 & 0.000 & 225.509 & 0.000 \\
K.PR & 0.414 & 0.002 & 27.020 & 0.018 \\
K.O & 0.570 & 0.000 & 32.224 & 0.006 \\
K.XM & -0.619 & 0.000 & -27.621 & 0.016 \\
K.YM & -0.465 & 0.001 & -15.612 & 0.146 \\
PR.O & 0.048 & 0.661 & 16.012 & 0.136 \\
PR.XM & -0.055 & 0.613 & -3.002 & 0.772 \\
PR.YM & 0.130 & 0.243 & -13.410 & 0.207 \\
O.XM & -0.178 & 0.118 & -13.810 & 0.195 \\
O.YM & -0.435 & 0.001 & -13.010 & 0.220 \\
XM.YM & 0.116 & 0.299 & 30.423 & 0.009 \\
\hline \hline
\end{tabular}

$$
\begin{aligned}
\text { Work }_{\text {physical }}= & 1.93+0.22 K+0.83 P R+0.110-0.80 X M+2.17 Y M+0.14 K^{2}+0.16 P R^{2} \\
& +0.18 O^{2}+2.07 X M^{2}+2.26 Y M^{2}+0.41 K * P R+0.57 K * O-0.62 K * X M \\
& -0.46 K * Y M+0.05 P R * O-0.06 P R * X M+0.13 P R * Y M-0.18 O * X M \\
& -0.44 O * Y M+0.12 X M * Y M
\end{aligned}
$$

Max-Force $e_{\text {physical }}$

$$
\begin{aligned}
& =67.37+21.35 K+36.56 P R+6.760-50.26 X M+142.51 Y M-16.27 K^{2} \\
& -9.07 P R^{2}-11.47 O^{2}+208.69 X M^{2}+225.51 Y M^{2}+27.02 K * P R+32.22 K \\
& * O-27.62 K * X M-15.61 K * Y M+16.01 P R * O-3.00 P R * X M-13.41 P R \\
& * Y M-13.81 O * X M-13.01 O * Y M+30.42 X M * Y M
\end{aligned}
$$




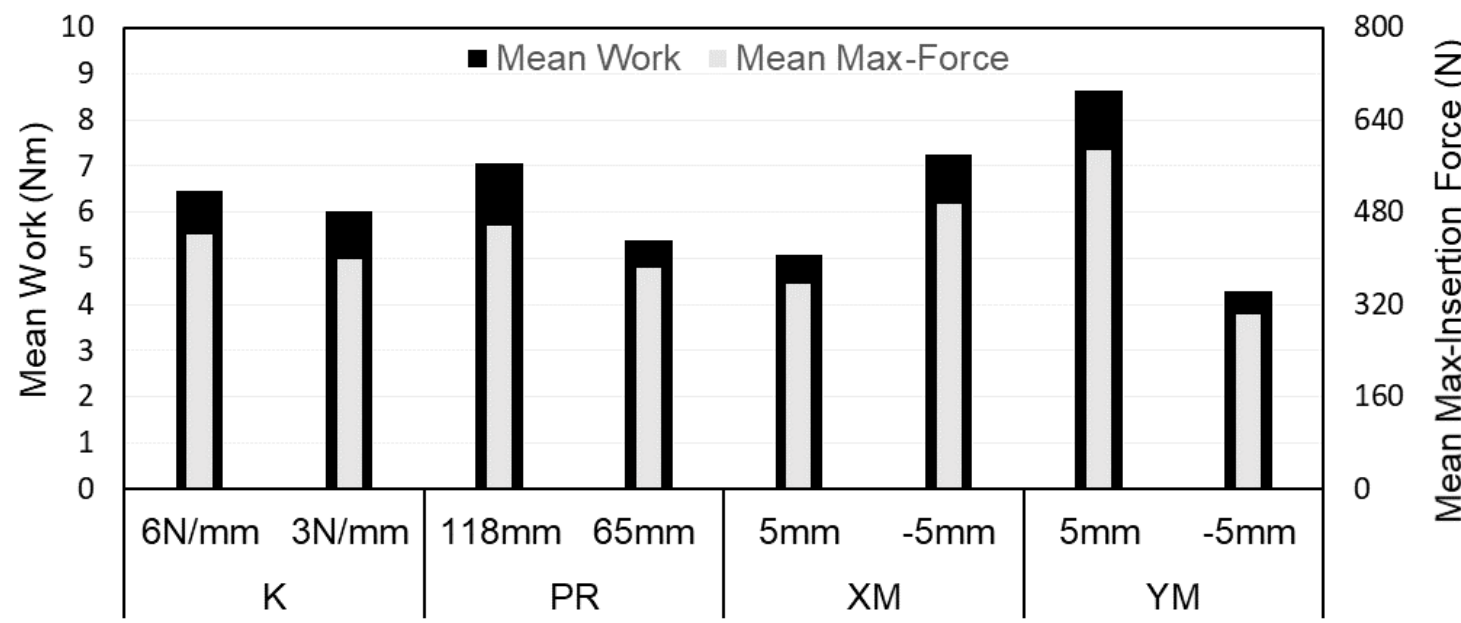

Fig. 9. Mean effect of controllable factors based on physical experiment data

Using the models presented as Eq. (11) and Eq. (12), end-effector responses can be predicted and visualised to provide insight into performance optimisation. Fig. 10 provides the effect of design factors $K$ and $P R$ on the maximum insertion force under varying misalignment conditions. Design factors $K$ and $P R$ reduce maximum force significantly. When using the softest springs, the average reduction is $43 \mathrm{~N}$, when pitch radius is minimum this value is $73 \mathrm{~N}$, and when both parameters are set to their lowest values, its 115 $\mathrm{N}$ with a maximum reduction of $170 \mathrm{~N}$ when $\mathrm{X}$-axis is misaligned $5 \mathrm{~mm}$ in the negative direction. This suggests that in order to reduce the maximum insertion force both $K$ and $P R$ should be set at -1 , which corresponds to the softest spring and smallest pitch radius. This in a sense provides opportunities to further improve the end-effector performance by using even softer springs and smaller pitch radius in future developments. 

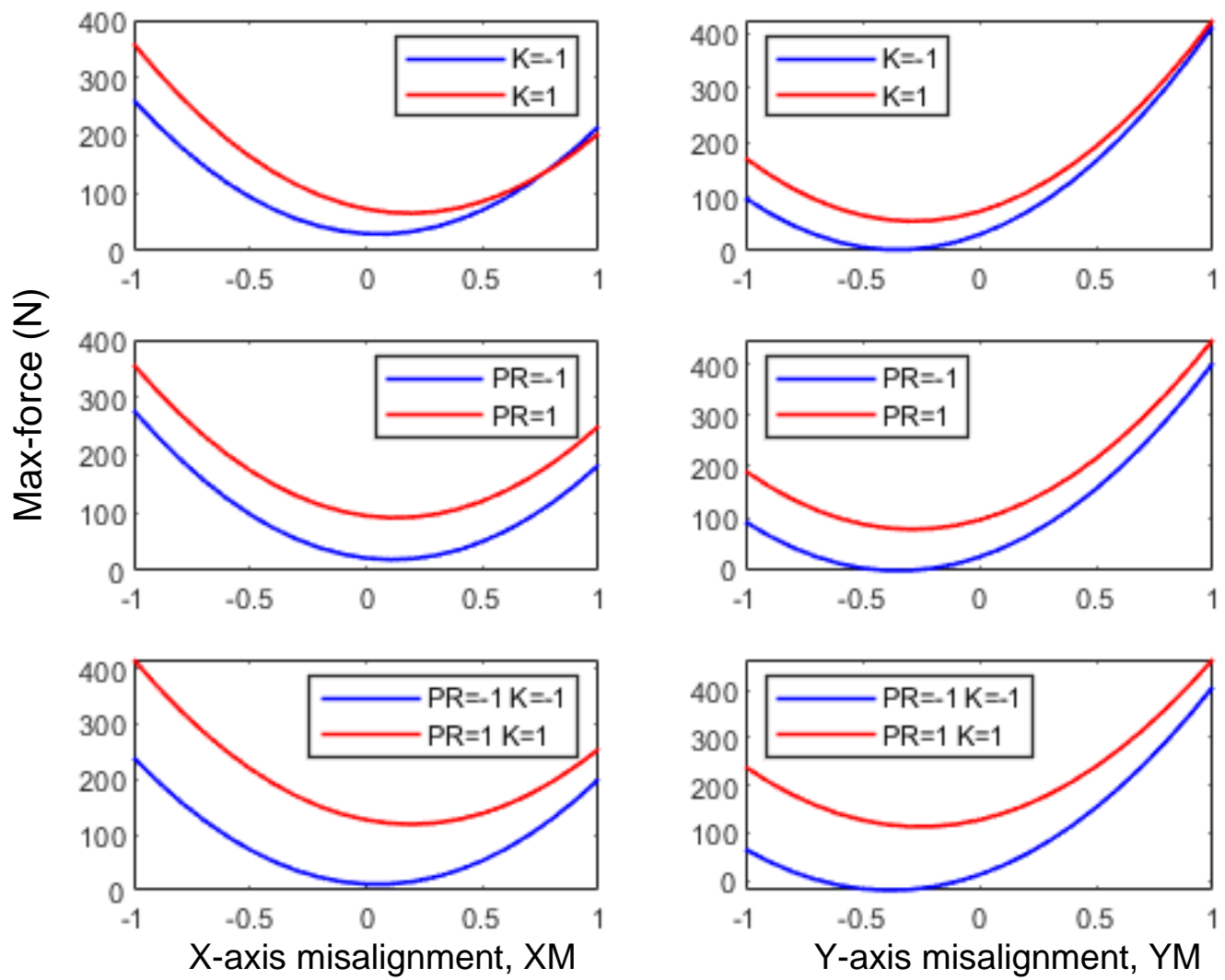

Fig. 10. Prediction of maximum insertion force with varying misalignment conditions: showing the effect of design factors on Maximum insertion Force.

\subsection{Simulation and calibration results}

Fig. 11 shows an example simulation result corresponding to run No. 10 in Table 6. Obvious differences can be observed from the figure suggesting the simulation model needs to be calibrated to reproduce the physical experiment outcome with reasonable accuracy.

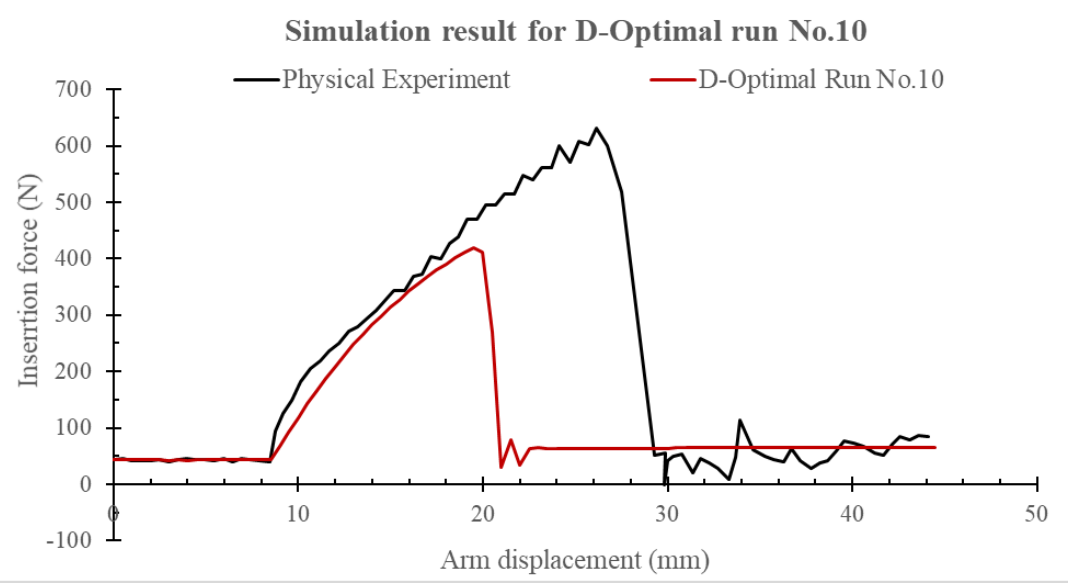

Fig. 11. An example simulation result plot (shown in red), with the physical experiment output shown in black. 
Table 6 shows the D-optimal results for work done and max-force calculated from the motion analysis output, compared to the results of physical experiment No.29. Two data columns, 'Work_diff' and 'Force_diff' are added to quantify the differences between simulation and physical experiment under various $F_{s}, P K$ and $Y K$ settings.

Table 6 Results for simulation for physical experiment No. 29.

\begin{tabular}{llllllllll}
\hline Order & Fs & PK & YK & $\begin{array}{l}\text { Work } \\
\text { physical }\end{array}$ & $\begin{array}{l}\text { Max-Force } \\
\text { physical }\end{array}$ & $\begin{array}{l}\text { Work } \\
\text { simulation }\end{array}$ & $\begin{array}{l}\text { Max-Force } \\
\text { simulation }\end{array}$ & Work_diff & Force_diff \\
\hline 1 & 0.26 & 550 & 550 & 9.34 & 630.87 & 8.58 & 517.63 & 0.76 & 113.24 \\
2 & 0.26 & 150 & 150 & 9.34 & 630.87 & 8.23 & 492.32 & 1.11 & 138.55 \\
3 & 0.06 & 150 & 550 & 9.34 & 630.87 & 3.11 & 303.29 & 6.23 & 327.58 \\
4 & 0.26 & 150 & 550 & 9.34 & 630.87 & 5.96 & 439.49 & 3.38 & 191.38 \\
5 & 0.26 & 350 & 350 & 9.34 & 630.87 & 6.82 & 479.49 & 2.52 & 151.38 \\
6 & 0.06 & 150 & 150 & 9.34 & 630.87 & 2.82 & 271.74 & 6.52 & 359.13 \\
7 & 0.06 & 550 & 550 & 9.34 & 630.87 & 2.87 & 242.74 & 6.47 & 388.13 \\
8 & 0.16 & 550 & 150 & 9.34 & 630.87 & 4.72 & 403.84 & 4.62 & 227.03 \\
9 & 0.06 & 550 & 350 & 9.34 & 630.87 & 2.28 & 243.06 & 7.06 & 387.81 \\
10 & 0.16 & 350 & 550 & 9.34 & 630.87 & 5.23 & 419.88 & 4.11 & 210.99 \\
11 & 0.06 & 350 & 150 & 9.34 & 630.87 & 2.72 & 260.14 & 6.62 & 370.73 \\
12 & 0.16 & 150 & 350 & 9.34 & 630.87 & 4.56 & 394.67 & 4.78 & 236.20 \\
13 & 0.26 & 550 & 150 & 9.34 & 630.87 & 8.59 & 524.16 & 0.75 & 106.71 \\
\hline \hline
\end{tabular}

Calibration of the simulation model is then achieved by exploring the most suitable settings for friction $\left(F_{s}\right)$, pitch compliance $(P K)$ and yaw compliance $(Y K)$ of the robot arm that yields the closest value to the outcome of the physical experiments. In other words, the optimal setting for $F_{s}, P K$ and $Y K$ should provide simulation results that are closest to the target values of $9.34\left(\mathrm{~T}_{1}\right)$ and $630.87\left(\mathrm{~T}_{2}\right)$ from the physical experiment selected. 'Work simulation' and 'Force $_{\text {simulation' }}$ results are fitted using full quadratic models whose statistics of fitted regression models can be found in Table 7. From this table, both models are seen to be significant with $\mathrm{p}$-values less than 0.05 and $\mathrm{R}^{2}$ close to 1 .

Table 7 Regression analysis results for the multi-objective minimisation

\begin{tabular}{llllll}
\hline \hline Model & $\mathrm{p}$-value & $\mathrm{R}^{2}$ & Adjusted R $^{2}$ & Root Mean Square Error & F-statistic \\
\hline Work $_{\text {simulation }}$ & 0.0117 & 0.987 & 0.946 & 0.543 & 24.5 \\
Max-Force $_{\text {simulation }}$ & 0.00357 & 0.994 & 0.976 & 16.6 & 55.1 \\
\hline \hline
\end{tabular}

Using the results from regression analysis, mathematical expression of the two models can be obtained, shown in Eq. (13) and Eq. (14).

$$
\begin{aligned}
& \text { Work }_{\text {simulation }}=4.66+2.48 F_{s}+0.19 P K-0.08 Y K+0.11 F_{s}^{2}-0.02 P K^{2}+0.50 Y K^{2}+0.51 F_{s} \\
& * P K-0.44 F_{S} * Y K+0.43 P K * Y K \\
& \text { Max-Force } \text { simulation } \\
& =405.94+113.69 F_{s}+1.63 P K-0.31 Y K-31.15 F_{s}^{2}-6.12 P K^{2}+8.79 Y K^{2} \\
& +26.06 F_{s} * P K-13.60 F_{s} * Y K+4.77 P K * Y K
\end{aligned}
$$

Eq. (13) and Eq. (14) are then tuned to meet the target values using Desirability functions (Costa et al., 2011). A nominal-the-best (NTB) desirability function is used in relation to these models such that a 
response that is either greater or less than the desired target values will reduce their desirability (Lee et al., 2018). Details of the applied desirability function is provided in Table 8.

Table 8 Desirability function settings for simulation model calibration

\begin{tabular}{|c|c|c|c|}
\hline Work $_{\text {simulation }}$ & $d_{1}=\left\{\begin{array}{cl}0, & y_{1}<L_{1} \\
\left(\frac{y_{1}-L_{1}}{T_{1}-L_{1}}\right)^{r_{1}}, & L_{1} \leq y_{1} \leq T_{1} \\
\left(\frac{U_{1}-y_{1}}{U_{1}-T_{1}}\right)^{r_{2}} & , T_{1} \leq y_{1} \leq U_{1} \\
0, & y_{1}>U_{1}\end{array}\right.$ & $\begin{array}{l}L_{1}=5 \\
T_{1}=9.34 \\
U_{1}=12 \\
r_{1}=1 \\
r_{2}=1\end{array}$ & (15) \\
\hline Max-Force simulation & $=\left\{\begin{array}{cl}0, & y_{2}<L_{2} \\
\left(\frac{y_{2}-L_{2}}{T_{2}-L_{2}}\right)^{r_{1}}, L_{2} \leq y_{2} \leq T_{2} \\
\left(\frac{U_{2}-y_{2}}{U_{2}-T_{2}}\right)^{r_{2}}, T_{2} \leq y_{2} \leq U_{2} \\
0, & y_{2}>U_{2}\end{array}\right.$ & $\begin{array}{l}L_{2}=500 \\
T_{2}=630.87 \\
U_{2}=1000 \\
r_{1}=1 \\
r_{2}=1\end{array}$ & $(16)$ \\
\hline Overall Desirability & $D=d_{1}^{0.5} \times d_{2}^{0.5}$ & & (17) \\
\hline
\end{tabular}

The optimal motion analysis settings of [ $\left.\begin{array}{lll}1 & 1 & -1\end{array}\right]$, whose actual factor levels can be found in in Table 9, is obtained from applying the desirability function. This table also provides the predicted simulation outcomes and its comparison with the physical results. It can be seen from the table that the optimal settings of $F_{s}, P K$ and $Y K$ is a point at the edge of the design space (see Fig. 12), indicating a better global optimum should exist outside the explored design space.

Table 9 Calibrated SolidWorks motion analysis settings

\begin{tabular}{lllllllll}
\hline \hline $\mathrm{F}_{\mathrm{s}}$ & PK & YK & Work $_{\text {simulation }}$ & MaxForce $_{\text {simulation }}$ & Work $_{\text {physical }}$ & Max & Work_diff & Force_diff \\
0.26 & 550 & 150 & 8.5191 & 527.988 & 9.34 & 630.87 & 0.821 & 102.882 \\
\hline \hline
\end{tabular}

Another optimisation approach, multi-objective minimisation using a Genetic Algorithm (Goldberg 1989) is applied for comparison. In this approach the responses need to be minimised meaning the optimisation towards specific work and max force values will not apply. As a result, the 'Work_diff' and 'Force_diff' are used as responses to be minimised. In other words, smaller differences indicate a closer match. The outcome of the multi-objective minimisation indicates [1, $0.99-1]$ for the motion analysis setting, which is almost identical to the results shown in Table 9.

The optimal motion analysis setting of $[0.26,550,150]$ is then implemented on the simulation model for physical experiment run No.29, the calibrated simulation outcome can be seen in Fig. 13. The forcedisplacement plot for the physical experiment and the example shown in Fig. 11 are also shown for comparison. A significant improvement is achieved compared to an uncalibrated model, which enables the reproduction of the coupling process with reasonable accuracy. Unlike the physical process in which vibrations are induced during coupling, hence the fluctuations, the simulation results exhibit a much smoother behaviour. 


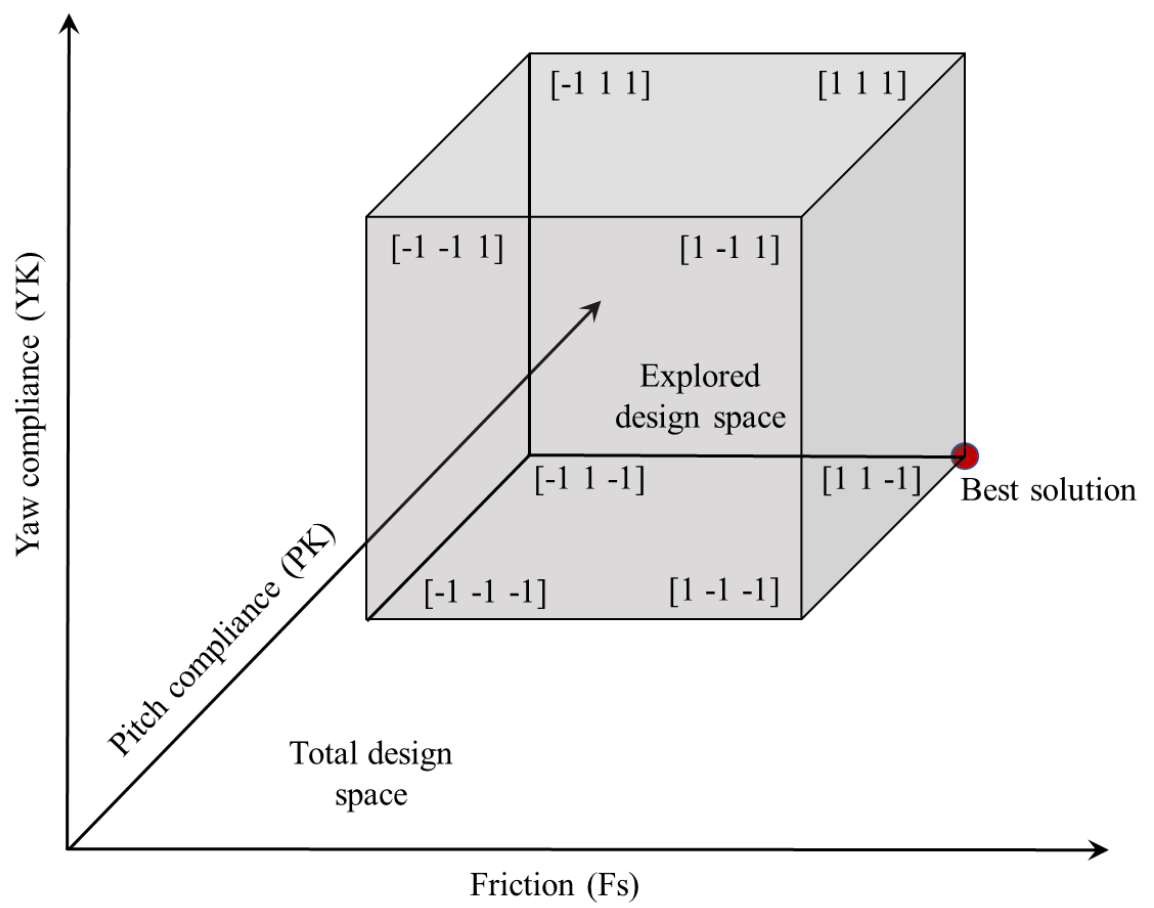

Fig. 12. Illustrates the best solution (for the calibrating the model) within the design space.

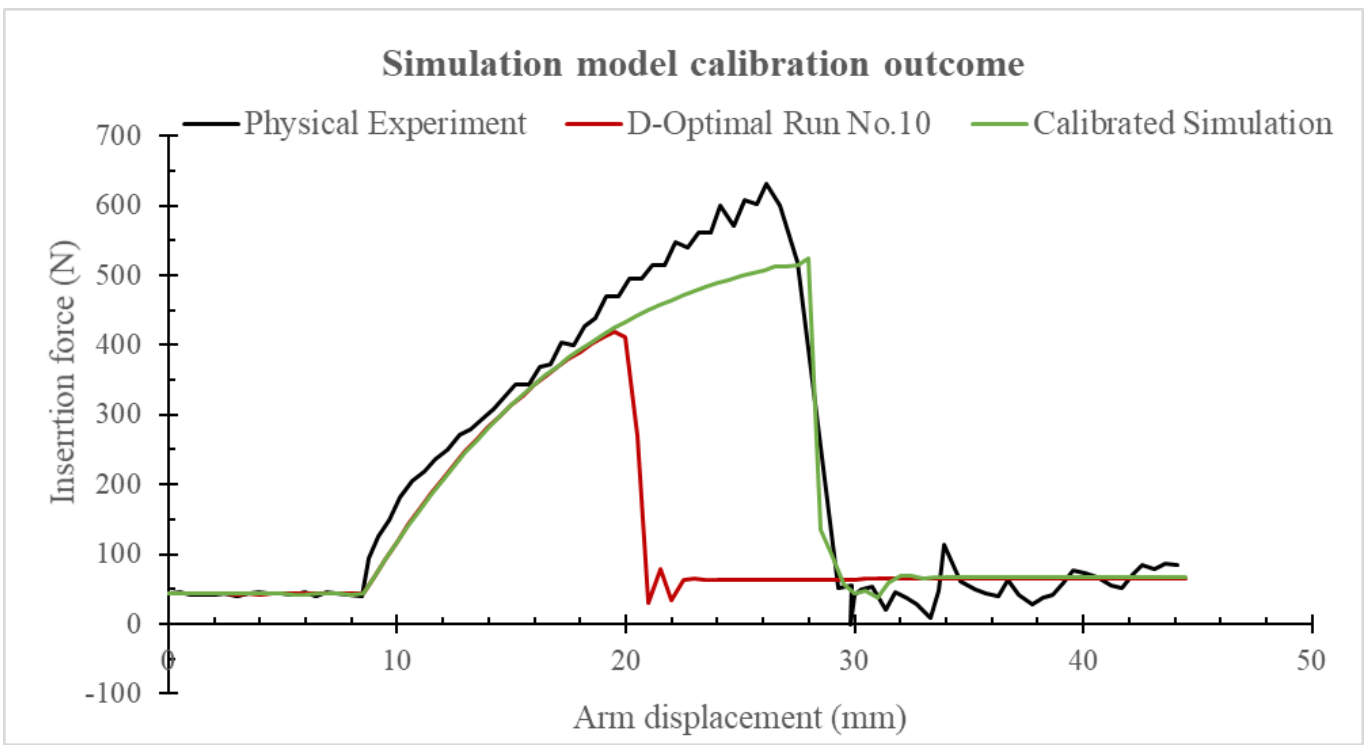

Fig. 13. Calibrated simulation results, shown in green, with physical experiment output shown in black and an example of uncalibrated simulation model shown in red (D-optimal run No.10).

In order to verify the optimal motion analysis settings obtained earlier, three additional simulations are performed corresponding to three physical experiments that are selected purposefully, together with No.29, to cover the four extreme conditions of misalignments. A summary of the calibration outcome for all four misalignment situations are shown in Table 10. 
Table 10 Verification of simulation model calibration

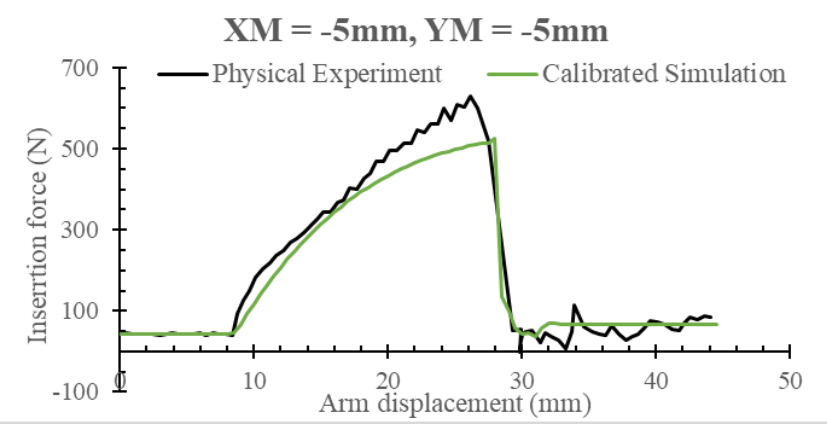

\begin{tabular}{|c|c|}
\hline Work (Physical) & 9.34 \\
\hline Max-Force (Physical) & 630.87 \\
\hline Work (Simulation) & 8.52 \\
\hline Max-Force (Simulation) & 527.99 \\
\hline Work (Difference in \%) & $9 \%$ \\
\hline Max-Force (Difference in \%) & $16 \%$ \\
\hline Work (Physical) & 9.03 \\
\hline Max-Force (Physical) & 650.09 \\
\hline Work (Simulation) & 7.75 \\
\hline Max-Force (Simulation) & 502.00 \\
\hline Work (Difference in \%) & $14 \%$ \\
\hline Max-Force (Difference in \%) & $23 \%$ \\
\hline Work (Physical) & 3.41 \\
\hline Max-Force (Physical) & 261.00 \\
\hline Work (Simulation) & 7.37 \\
\hline Max-Force (Simulation) & 439.02 \\
\hline Work (Difference in \%) & $116 \%$ \\
\hline Max-Force (Difference in \%) & $68 \%$ \\
\hline Work (Physical) & 9.30 \\
\hline Max-Force (Physical) & 570.03 \\
\hline Work (Simulation) & 8.81 \\
\hline Max-Force (Simulation) & 466.81 \\
\hline Work (Difference in \%) & $5 \%$ \\
\hline Max-Force (Difference in \%) & $18 \%$ \\
\hline
\end{tabular}


From the figures in Table 10 it is observed that three out of four verifications show reasonable accuracy when reproducing the coupling process considering the local optimal setting applied. The difference in percentage shown in the table provides a quantitative measure. Apart from the third verification where XM $=5 \mathrm{~mm}$ and $\mathrm{YM}=-5 \mathrm{~mm}$, all other results produced by the simulation models are generally smaller than what was obtained from physical experiments, which again indicates a better global optimum outside the design space investigated. For the third verification, a noticeably large difference between simulation and physical results is observed, this indicates that when XM is $5 \mathrm{~mm}$ and $\mathrm{YM}$ is $-5 \mathrm{~mm}$, the calibrated simulation model should not be used to represent the physical experiments.

\section{Discussion}

A benefit of having passive compliance in a robot end-effector is that it will help to improve the robustness of fluid coupling whilst reducing the reliance on accurate robot end-effector positioning systems. In this work, the role of end-effector compliance in enhancing the mechanical connection of fluid ports under positional uncertainties was investigated in both physical and numerical environments. Results from the physical experiments show that misalignments have an exponential effect on the work done and the maximum force of insertion. When the fluid port is misaligned, having softer springs at a lower pitch radius can reduce the maximum insertion force by up to $160 \mathrm{~N}$. Likewise, work done by the end-effector is reduced indicating a better coupling. When there are no misalignments, a force of about $43 \mathrm{~N}$ is required to drive the robot arm at the nominal speed. Forces observed beyond this value are due to contact friction and physical clash. When the port is misaligned $5 \mathrm{~mm}$ in the parallel direction of $\mathrm{X}$ and $\mathrm{Y}$, the maximum insertion force is around $660 \mathrm{~N}$. This value is considered large when compared to the typical force exerted manually by a human. In addition, current train fluid ports are not designed for such loads that also require larger robot motors and a stronger structure supporting the fluid port.

As discussed above, further improvement is required in the performance of the end-effector. However, if the end-effector design concept changes, a completely physical approach requires manufacture of the new end-effector, further experiments and analysis. Such efforts are not encountered if the development process follows a numerical approach. However, as it was shown, a direct numerical approach will not accurately capture the complex reality, such as geometrical, material and boundary condition imperfections. The hybrid approach captures such effects and represents them in the simulation model, where robot compliance and friction imperfections have played a significant role in calibrating the simulation models.

The results indicate that the true optimum of the calibrated robot compliance and friction factors lie outside of the design space explored by the designated DoE plan. This indicates that the study should have incorporated a broader range of factor values. Nonetheless the desirability function employed has indicated a local optimum which has certainly enhanced the simulation model. Error of up to $16 \%$ was observed when matching the physical results used to calibrate the model. As was shown in the results of Section 4.2, this is significant improvement. However, this accuracy reduces when applied to some other physical conditions such as different misalignment cases. In this regard, the DoE and analysis of the physical experiments can provide further insight.

Considering the compliances of the end-effector for instance, it is not symmetrical in pitch motions. When the coupler pivots downwards it only deforms one spring, and when it pivots upwards it will deform two springs. Thus, as seen from the physical results, pivoting downwards due to a corresponding misalignment requires less work or force. By design, the end-effector should have symmetrical compliance in yaw motions but results also show a lack of symmetry where positive misalignment tend to be easier to overcome. Thus, it is clear that inherent robot compliance, backlash and other hysteresis create preferred directions of motion. These effects were not well captured by the linear torsional springs employed in the numerical model, as these springs behave similarly in both directions of motion (clockwise/anticlockwise). Consequently, the accuracy of the calibrated model will alter when applied to different robot and misalignment configurations. In order to address this, more factors can be added to the simulation calibration DoE plan with the aim of capturing such effects. For example, a nonlinear torsional spring with 
custom force-displacement relationship can be used to test different compliances in different directions of motion. By increasing the experiment size, i.e. number of runs, more accurate regression models can be obtained hence the coupling performance can be predicted more accurately. Similarly, more experiment runs enable regression models with higher order to be obtained which potentially could also improve the accuracy predicted coupling performance. Consideration of new factors can reveal more complex relationships between those factors and coupling performance. Therefore, a second iteration of experiments with new factors and modified factor ranges should reveal a new and reachable better optimum.

An advantage of this hybrid approach lies in less effort in obtaining results in a simulation environment when reality is well represented. Reliance on physical experiments will be reduced in future testing and evaluation of end-effectors by virtue of the physical-numerical hybrid approach that has been presented. Once the calibrated numerical model has an acceptable accuracy it is then possible to investigate other compliance designs for the end-effector. However, a major limitation of the model is that the fluid couplers should be the only parts in contact between the robot and train. When using the calibrated simulation model, any new end-effector analysis should follow this rule.

It is worth noting that SolidWorks Motion Analysis force expressions are a very powerful tool for representing any nonlinear compliance during motion analysis simulations. This requires the forcedisplacement relationship of the element or mechanism to be represented. However, with recent developments in the field of compliant mechanism design, deriving such expressions should not be a difficult task. This is even true for large motion compliant mechanisms where PRBM can be used for deriving the expression.

\section{Conclusion}

Physical experimental results on the CyberFluids robot train fluid port demonstrator indicated an improvement in reducing maximum end-effector insertion force by up to $170 \mathrm{~N}$ in misaligned fluid port couplings. The effects of robot compliance were also revealed and found to be asymmetric, leading to offset insertion forces for different misalignment directions. Nevertheless, forces observed during the insertion are still too large for practical applications hence, this research will continue beyond this preliminary study to seek better design of end-effector compliance. In order to facilitate future progress, a hybrid, numericalphysical approach was proposed to construct and calibrate a numerical model, which in practice aims to reduce future efforts and physical labour in testing of end-effectors. It was shown that the characteristic curve for insertion force obtained from the calibrated simulations can be representative of the physical measurements. This illustrates the model capability in compensating for imperfections and subtleties that exist in the physical reality. In further detail, the DoE and desirability method used to determine the optimal settings of the model has reduced simulation error down to $16 \%$ for maximum insertion force, and $9 \%$ for work done. This is significant improvement considering the true optimum settings of the model may be outside the explored design space, and more calibration factors can be added to further enhance the model. When the calibrated model was tested for other robot and misalignment configurations, the simulation results showed greater error, suggesting that the model should only be used for the calibrated configuration. This can be resolved by expansion of the calibration DoE and using more physical scenarios as benchmarks for the calibration.

\section{Acknowledgments}

We especially thank and acknowledge the Rail Safety and Standards Board (RSSB) for funding and supporting this research (contract RSSB 2675). 


\section{References}

Asada, H, \& Kakumoto, Y. (1988). The dynamic RCC hand for high-speed assembly. IEEE Int Conf Robot Autom, April 24-29 1988 (pp 120-125). Philadelphia, PA, USA, USA.

Atherton, M. A. \& Bates, R. A. (2006). Searching for improvement. Design and Information in Biology, 27, 345-379.

Atherton, M., Hill, S., Harrison, D., \& Ajovalasit, M. (2019). Economic and technical feasibility of a robotic autonomous system for train fluid servicing. Journal of Rail Rapid Transit, 234(3) 338-350.

Costa, N.R., Lourenço, J., \& Pereira, Z. L. (2011). Desirability function approach: A review and performance evaluation in adverse conditions. Chemometrics and Intelligent Laboratory Systems, 107(2), 234-244.

Dassault Systemes. (2020). Retrieved September 25, 2020, from https://www.solidworks.com/

Dixon company (2020). Retrieved September 25, 2020, from https://europe.dixonvalve.com/en-gb

Eshraghi, K., Jiang, P., Suraci, D., \& Atherton, M. (2020). Preliminary study on end-effector compliance in automated fluid coupling for trains. In I. Horváth, \& G. Keenaghan (Ed.), Proceedings of TMCE 2020, (pp. 205-216). Dublin.

Goldberg, D.E. (1989), Genetic Algorithms in search, optimization, and machine learning, Addison Wesley Longman.

Haskiya, W., Maycock, K. and Knight, J. (1998). A passive compliant wrist for chamferless peg-in-hole assembly operation from vertical and horizontal directions. Proceedings of the Institution of Mechanical Engineers, Part B: Journal of Engineering Manufacture, 212(6), 473-478.

Hawkes, E.W., \& Cutkosky, M.R. (2018). Design of materials and mechanisms for responsive robots., Annual Review of Control, Robotics, and Autonomous Systems, 1, 359-384.

Help.solidworks.com. 2020. Motion Analysis Overview. [online] Available at: <https://help.solidworks.com/2020/English/SolidWorks/motionstudies/c_Motion_Analysis.htm> [Accessed 10 July 2021].

Hinkelmann, K. (2012). Design and Analysis of Experiments. (5th ed.). USA

Jiang, J., Bian, C., \& Ke, Y. (2017). A new method for automatic shaft-hole assembly of aircraft components. Assembly Automation, 37(1), 64-70.

Kamnik, R., Rodič, G., Mihelj, M. and Bajd, T., 2001. Automation of the car battery lid assembly operation. Robotics and Computer-Integrated Manufacturing, 17(6), 435-446.

Lee, D.H., Jeong, I.J., \& Kim, K.J. (2018). A desirability function method for optimizing mean and variability of multiple responses using a posterior preference articulation approach. Quality and Reliability Engineering International, 34(3), 360-376.

Montgomery, D., (2012). Design and analysis of experiments. 8th ed. John Wiley \& Sons Inc.

Myers R. H. (1999). Response surface methodology - current status and future directions. Journal of Quality Technology, 31(1), 30-44.

Pitchandi, N., Subramanian, S. and Irulappan, M., (2017). Insertion force analysis of compliantly supported peg-in-hole assembly. Assembly Automation, 37(3), pp.285-295.

Rolling Stock Strategy Steering Group. (2018). Long Term Passenger Rolling Stock Strategy for the Rail Industry. Retrieved September 25 2020, from

https://www.raildeliverygroup.com/files/Publications/2018_3_long_term_passenger_rolling_stock_s trategy_6th_ed.pdf

Sathirakul, K. \& Sturges, R. H. (1998). Jamming conditions for multiple peg-in-hole assemblies, Robotica 16, 329-345.

Shigley, J. (2006). Standard handbook of machine design. (2nd ed). Norwich, NY: Knovel. 
Help.solidworks.com. 2021. Motion Analysis Overview - 2020. [online] Available at: <https://help.solidworks.com/2020/English/SolidWorks/motionstudies/c_Motion_Analysis.htm> [Accessed 31 July 2021].

Sturges, R. \& Laowattana, S., (1996). Design of an Orthogonal Compliance for Polygonal Peg Insertion. Journal of Mechanical Design, 118(1), p.106.

Wang, S., Chen. G., Xu, H., \& Wang. Z. (2019). A robotic peg-in-hole assembly strategy based on variable compliance center. IEEE Access, 7, 167534 - 167546. doi: 10.1109/ACCESS.2019.2954459.

Wang, W., Loh, R., \& GU, E. (1998). Passive compliance versus active compliance in robot-based automated assembly systems. Industrial Robot: An International Journal, 25(1), 48-57.

Whitney D.E. (1982), Quasi-Static Assembly of Compliantly Supported Rigid Parts. ASME.

J. Dyn. Sys., Meas., Control.104(1):65-77.

Xu, J. Hou, Z. Liu, Z \& Qiao, H. (2019). Compare Contact Model-based Control and Contact Model-free Learning: A Survey of Robotic Peg-in-hole Assembly Strategies. arXiv:1904.05240 [cs], April 2019. Retrieved from http://arxiv.org/abs/1904.05240.

Zohoor, H. \& Shahinpoor, M., (1991). Dynamic analysis of peg-in-hole insertion for manufacturing automation. Journal of Manufacturing Systems, 10(2), 99-108.

\section{Author Biographies}

Kourosh Eshraghi is a Doctoral Researcher in the Department of Mechanical and Aerospace Engineering, Brunel University London. His research field is compliant mechanisms.

Pingfei Jiang is a Research Fellow in the Department of Mechanical and Aerospace Engineering, Brunel University London. His research fields are data-driven design and design innovation.

Daniele Suraci is a Visiting Research Fellow in the Department of Mechanical and Aerospace Engineering, Brunel University London. His research field is the design of autonomous systems.

Mark A. Atherton is Professor of Mechanical Engineering Design in the Department of Mechanical and Aerospace Engineering, Brunel University London. His research fields are robust engineering design and design innovation. 